\title{
Primary Health Care and leprosy
}

\author{
T P CULLINAN \\ 53 Mapledene Road, Dalston, London E8
}

Received for publication 31 December 1981

\section{Principles}

It is becoming increasingly clear that simple and universal solutions to the control of leprosy are inadequate, and great efforts are being made to understand not only its medical characteristics but also the social, economic and cultural factors that make its control so difficult. Approximately 15 million people suffer from leprosy today and there is little evidence that the number is decreasing; the annual incidence is around $\frac{3}{4}$ million. ${ }^{1}$ Despite the technological means to do so, the disease still eludes control, even in those countries which can afford it, due to a number of problems concerning case detection, case holding, drug treatment and follow-up. It is a discussion of these problems in relation to Primary Health Care (PHC) that provides the main theme of this essay.

In what must appear to many countries a verbose report based on dreams, the Alma-Ata Conference declared that 'PHC is the key to achieving an acceptable level of health throughout the world'. ${ }^{2}$ Essentially, PHC is simply the care available at the first point of contact between the patient, or the patientto-be, and the health services. This bald statement says little of the true complexity of the situation. Firstly, a primary health system that is appropriate in one country, or even one area, may be entirely inappropriate in another. To be useful, it must be accepted, and so must be sensitive to the cultural, economic, intellectual, technological and even medical idioms of the society it serves. If, of necessity, planning must sometimes be aided by distant experts, operation should always be in local hands. 'A guiding principle is that PHC is first and foremost a community effort. ${ }^{3}$ This is probably nowhere more true than in a

* This article is an edited and abridged version of a prize-winning essay submitted by the author for the 1981 LEPRA Prize Essay Competition, which is offered annually to all medical schools in the United Kingdom. Editor. 
disease such as leprosy which is so deeply embedded in local consciousness, and in which standard methods of treatment are so lengthy. A community health initiative of this kind may involve a radical reconsideration of some firmly held precepts, including that by which new medical knowledge is allowed to originate only in the rich countries and to flow, often unsuitably, to the Third World periphery.

It is important that a primary health system must somehow be integrated into, and remain in step with other economic and social developments. It has a social significance that never emerged from the more traditional, vertical health systems. ${ }^{4}$ As a recent conference of the 31 poorest nations demonstrated, a large number of them (mostly with significant leprosy problems) are going to remain extremely poor in the foreseeable future and it is thus vital that the medical services are realistically planned to be both effective and economic.

The first practical task of a government organizing a primary health programme is the definition of its objectives. If these are considered with realism and with some exactitude, they will serve well as indices for later evaluation of the scheme. For leprosy, complete eradication is probably not possible ${ }^{5}$ and a more practical objective for a primary health programme for leprosy is its control. At present, the control of this infection requires the careful and individual chemotherapy of all patients with active disease to a level sufficient to eliminate the reservoir of mycobacteria, but there are some basic flaws in its principle which are largely responsible for the failure, to date, of many programmes. When dapsone was first discovered to be an effective antileprous drug in $1943^{6}$ and increasingly used over the next $10-15$ years, it was thought to be an easy and effective way to control the disease. Mass medication of patients would lead to the eventual eradication of leprosy. A brief look at the prevalence of the disease today shows that such hopes have not been realized. The mere possession of an effective cure does not guarantee its effective distribution - leprosy (like tuberculosis) provides a fine example of the 'application gap'. ${ }^{7}$

Implicit in the objective of leprosy control is a means of monitoring this control, and it is worth considering what contribution can be made by workers at the periphery. In some circumstances, prevalence rates may be measurable by field workers whose basic education is often rudimentary. In the report of the first conference on epidemiological techniques for primary care schemes, ${ }^{8}$ it was stated (perhaps somewhat surprisingly) that 'front-line' health workers, in general, adopted simple survey methods with comparative ease. However, difficulties remain. The lack of notification systems and national records together with population mobility and confusion over names and addresses make repeated sample framing and follow-up very difficult. However, Copplestone ${ }^{9}$ has emphasized the importance in these situations, of accepting inevitable inaccuracies and allowing for them, usually by overestimation. There are, furthermore, some methods whereby a village health worker, under careful 
supervision, may carry out meaningful prevalence sampling, in selected areas. For example he may examine all people along certain randomly chosen compass directions, from a central point in the village. This method requires no more equipment than a compass and the writing of some simple records. Repeated observations of samples such as these may provide a series of local prevalence rates which will help to measure the effectiveness of the control programme. However the problems should not be underestimated; according to $\mathrm{WHO}^{10}$ attempts by single workers to survey populations in S.E. Asia have been disappointing and even misleading. The application of these methods to urban areas has been doubted by Mutalker ${ }^{11}$ who pointed out that house to house detecting in Poona was impossible because of the community's attitudes to 'private life and other living conditions'.

At the hub of primary care is the village -.. or urban - community worker. In the leprosy field, his (or her) responsibilities will lie in case-finding and referral for diagnosis, the supervision of treatment and attendance for supervised drugs, rudimentary rehabilitation, and health education. The latter may be of particular importance. Local feelings about leprosy must never be underestimated if only because deeply held beliefs may rub contrary to received medical opinion and thus hamper control. Although the importance of health education is undeniable, effective methods are harder to define. Obviously they must be appropriate to the 'target' and easily applied. In Bombay, intensive radio coverage, following popular programmes, has been combined with a school essay competition (in which 100,000 children take part each year!) with apparently good results. ${ }^{12}$ In communities with fewer resources, and particularly in rural areas, less sophisticated methods will be appropriate and many of these may revolve around the local school. Whilst peripheral health care workers must be encouraged to detect and refer all leprosy cases, their role in the early (or earlier) detection of multibacillary cases may be crucial to the effectiveness of the control programme, and their potential in the early recognition of minimal changes on the skin surface may be considerable.

However, the community health worker should not become merely a 'leprosy worker'. The primary control of leprosy must not run parallel to the main body of PHC but should be integrated, as far as possible, into existing health services. According to Chwatt, ${ }^{13}$ a significant factor in the failure of malaria control over the last 20 years, has been the 'vertical, target-orientated, mass campaign' approach to the problem. Such campaigns directed from outside on to a particular disease, have the attraction of impetus and immediacy, and may offer some degree of early success; they also enjoy an air of excitement that may be lacking in the more prosaic and dogged 'horizontal approach'. Leprosy is a chronic and potentially crippling disease which affects large numbers of people spread over wide areas and in many countries it is likely that the best approach to leprosy control will be through local and comprehensive health service; this also has the advantage of reducing the stigma of 
leprosy which its isolated management can only enhance. Browne ${ }^{14}$ emphasizes that wherever possible, only existing diagnostic and treatment facilities should be used.

The training of the community worker, normally at the primary hospital, should be reinforced by frequent 'refresher' courses and should also involve regular visits of those at hospital level to the communities. Not only does this help to maintain standards and morale (a very important feature) but it also helps to remind hospital staff of the problems at village level. The importance of repeated personal contact and supervision, particularly in a rather slowmoving disease such as leprosy, cannot be overestimated. Without it, the system may founder.

\section{Practice}

Although WHO, in its Technical Report of $1977,{ }^{1}$ has stated that 'after a reasonable period of training', community health workers are generally capable of a sound diagnosis on clinical grounds alone, the present understanding of the term 'PHC worker' suggests very strongly indeed that he should not be concerned with the diagnosis of leprosy - but only with the detection and recognition of suspected cases. These should then be referred to a trained observer for confirmation of diagnosis before treatment is started.

Once the patient has been confirmed as having leprosy and classified as to type, the peripheral worker may be able to contribute greatly to regularity of drug intake and attendance. Although chemotherapy will not alone control leprosy, it is the main tool available for this purpose. Leprosy control by this tool is however plagued by the twin problems of poor patient drug compliance and irregular attendance at out-patient clinics. It may be that it is simply the chronic nature of leprosy, and its necessarily prolonged treatment, that highlights these problems, but it is likely that the aura built up around the disease over the centuries is also an important factor, since the patient has to 'recognize' the disease in himself and often to 'reveal' it to his family, neighbours and perhaps employers. Regular, kindly interest and encouragement by a locally based worker in village, community or slum may be of enormous value.

So complex are the problems of mass chemotherapy, that the search for other tools of control, which may be implemented by a primary type scheme, seems essential. A preventative approach is an obvious choice. WHO does not recommend either prophylactic dapsone or BCG vaccinations, which are both of dubious value, the first merely compounding the problems outlined above. However, a vaccine, offering active immunization, is far more promising and even at this early stage it may not be entirely out of place to consider the possibility that PHC workers may be called upon to participate in the administration of such a vaccine to the enormous numbers of people who may be at 
risk in endemic areas. Indeed some authorities have doubted if a vaccine could be administered to a satisfactory number of people with existing health systems.

The subjects of chemotherapy and prevention must not divert primary health service attention entirely from the crippling effects of this disease. To the sufferer these are all-important and the primary health worker may be as busy with the disabled as with the care of newly diagnosed patients. Although some disabled patients will need referral to hospital for skilled physiotherapy, prostheses or reconstructive surgery, certain techniques are open to the primary level worker; for example, simple eye exercises for lagophthalmos, provision and repair of simple protective footwear for denervated feet, hand exercises and constant education on the care of anaesthetic hands and feet.

Much of the above work requires the primary health worker to keep simple records. The form and sophistication of these will obviously vary with the community worker's ability and the objectives of the local leprosy programme. It is regrettable that this subject has been hardly studied; in the only paper devoted exclusively to leprosy records, ${ }^{15}$ no mention at all is made of primary level workers. Most primary workers have a degree of literacy which would allow simple written records and these could be augmented by abacus-type systems, as are traditionally used in some Central African countries or by forms which make use of pictures and line drawings of relevant subjects.

The finance allocated to a primary leprosy control programme reflects the priority allocated to it by central government. ${ }^{16}$ Most countries where leprosy is endemic are poor and likely to remain so for the near future. In a detailed paper, Lechat and others have analysed the costs of various methods of controlling leprosy. ${ }^{17}$ As expected, a programme of vaccination (for all children and those in hyperendemic areas) is far the most effective method of control, though requiring a large capital outlay. This sum could probably be reduced by carefully integrating the leprosy vaccination scheme into other mass-vaccination programmes. The present method of control - the early detection of cases and their prolonged treatment -- is considerably more expensive, and so far rather less effective. Ten years ago, the LEPRA project in Malawi was estimated to cost $£ 3.10$ per patient per year. ${ }^{17} \mathrm{~A}$ breakdown of such expenses would show that the major part of the budget of many programmes is spent on (a) the necessarily prolonged treatment and supervision of patients, and (b) transport and drug delivery costs. (These have increased enormously over the past years.) Major reductions in these areas could be made by the earlier detection of cases, so reducing the likelihood of disabilities and their subsequent care, and by the determined application of combined chemotherapy for pauci- and multibacillary cases, as recently recommended by WHO. 


\section{Conclusion}

As a principle, the primary health approach to the control of leprosy has obvious advantages. It is 'appropriate', cheap, durable and available to those who need it most. It is firmly rooted in the community, and incorporates the best principles of preventive and educative medicine. Above all, it places the control of leprosy in a broad schema of illness and health, related to socioeconomic conditions. Yet there has so far been a failure, admittedly not unique to leprosy, to apply this model to the reality of the disease. Leprosy is as common today as it was 20 years ago.

This is not through lack of effort or ignorance. Though much of it is little more than pontification, there is an extensive literature on the principles and philosophy of PHC community involvement and comprehensive health care. Much knowledge has also accumulated on the practical aspects of leprosy control in the field. It is in the gap between such principles and the practice of leprosy control that the problem lies. What is needed is an approach that is sufficiently imaginative, broad and 'visionary' to bridge it.

\section{References}

1 WHO Expert Committee on Leprosy: Fifth Report. Technical Report Series, 1977, 607.

${ }^{2}$ Report of the International Conference of Primary Health Care Alma-Ata 1978. WHO Geneva.

${ }^{3}$ Kawaluddin M. President of 31 st World Health Assembly. WHO Chronicle, 1978: 32(11): WHO Geneva.

${ }^{4}$ Quenum C. Health for all by the year 2000 as a revolutionary concept. World Health Forum, 1981;2(7): 105 .

Warren K. Tropical Medicine from Romance to Reality. Academic Press London, 1978.

${ }^{6}$ Faget, Pogge et al. The promin treatment of leprosy 1943. Public Health Reports (Washington), 58: 1729.

7 Editorial Lancet 1976; ii: 615.

8 WHO Document SHS/79.2 in WHO Chronicle, 1980; 34(1): WHO Geneva.

${ }^{9}$ Copplestone. Planning an epidemiological field survey. WHO Chronicle 1965; 29(6): WHO Geneva.

10 Guide to Leprosy Control. 1980: WHO Geneva.

11 Mutalker. Health education in leprosy. Leprosy in India, 1977; 49 (2).

12 Antia. The people we fail to reach. Editorial Lep Rev, 1977;48: 155.

${ }^{13}$ Chwatt B. The challenge of malaria - tropical medicine: In: Wood C. ed. From Romance to Reality. Academic Press, London, 1978.

14 Bryant J. Community health workers. WHO Chronicle, 1978; 32(4): Geneva.

15 Mogli G. Medical records in leprosy programme. Leprosy in India, 1979; 51 (2).

${ }^{16}$ Samba EM. Primary Health Care - unknowns, pitfalls and hazards. World Health Forum, 1981;2(3): Geneva.

17 Molesworth B. LEPRA control project in Malawi. Lep Rev, 1969; 40: 237. 